\title{
Impact of the Halide Cage on the Electronic Properties of Fully Inorganic Cesium Lead Halide Perovskites
}

\author{
Z. Yang, $^{\dagger}$ A. Surrente, ${ }^{\dagger}$ K. Galkowski, ${ }^{\dagger,}$ A. Miyata, ${ }^{\dagger}$ O. Portugall, $^{\dagger}$ R. J. Sutton, ${ }^{\text {I॰ }}$ A. A. Haghighirad, ${ }^{q}$ \\ H. J. Snaith, ${ }^{\text {II }}$ D. K. Maude, ${ }^{\dagger}$ P. Plochocka, ${ }^{* \dagger}{ }^{\dagger}$ and R. J. Nicholas*, ${ }^{*}$
}

${ }^{\dagger}$ Laboratoire National des Champs Magnétiques Intenses, CNRS-UGA-UPS-INSA, 143 avenue de Rangueil, 31400 Toulouse, France

${ }^{\ddagger}$ Institute of Experimental Physics, Faculty of Physics, University of Warsaw, Pasteura 5, 02-093 Warsaw, Poland

${ }^{\text {II }}$ Clarendon Laboratory, University of Oxford, Parks Road, Oxford OX1 3PU, United Kingdom

\author{
Supporting Information
}

ABSTRACT: Perovskite solar cells with record power conversion efficiency are fabricated by alloying both hybrid and fully inorganic compounds. While the basic electronic properties of the hybrid perovskites are now well understood, key electronic parameters for solar cell performance, such as the exciton binding energy of fully inorganic perovskites, are still unknown. By performing magnetotransmission measurements, we determine with high accuracy the exciton binding energy and reduced mass of fully inorganic $\mathrm{CsPbX}_{3}$ perovskites ( $\mathrm{X}=\mathrm{I}, \mathrm{Br}$, and an alloy of these). The well-behaved (continuous) evolution of the band gap with temperature in the range of 4-270 K suggests that fully inorganic perovskites do not undergo structural phase transitions like their hybrid counterparts. The experimentally determined dielectric constants indicate that at low

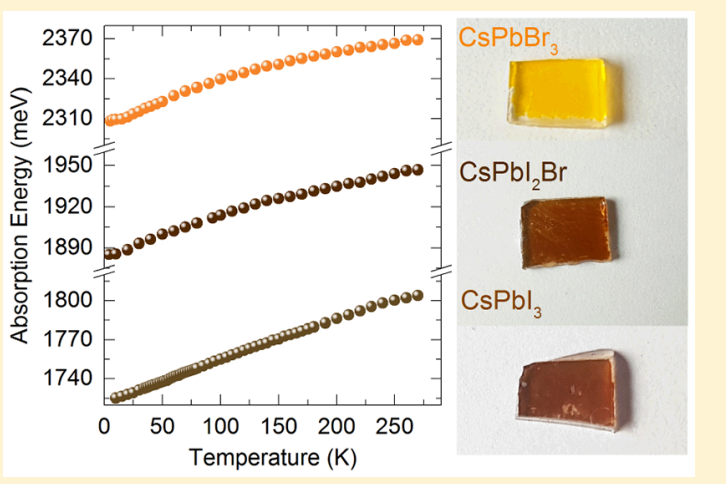
temperature, when the motion of the organic cation is frozen, the dielectric screening mechanism is essentially the same for both hybrid and inorganic perovskites and is dominated by the relative motion of atoms within the lead halide cage.

$\mathrm{R}$ apid developments in the field of hybrid organicinorganic perovskites have led to a dramatic increase of power conversion efficiencies in perovskite-based solar cells, which currently exceed $22 \% .^{1-4}$ Hybrid organicinorganic perovskites combine low-cost fabrication processes 5,6 with strong light absorption, ${ }^{7}$ efficient photoluminescence, ${ }^{8,9}$ together with long carrier lifetimes and diffusion lengths. ${ }^{10,11}$ The combination of these properties has led to numerous applications of this class of materials in optoelectronic devices beyond solar cells, including light-emitting diodes, ${ }^{12}$ lasers, ${ }^{13}$ and photodetectors. ${ }^{14}$

Hybrid organic-inorganic perovskites are characterized by a general chemical formula $\mathrm{ABX}_{3}$, where $\mathrm{A}$ is an organic ammonium cation (methylammonium (MA), or formamidinium (FA) ), $\mathrm{B}=\mathrm{Pb}^{2+}$ or $\mathrm{Sn}^{2+}$, and $\mathrm{X}$ is a halide anion $\left(\mathrm{Cl}^{-}\right.$, $\mathrm{Br}^{-}, \mathrm{I}^{-}$, or an alloyed combination of these). Initially, the fabrication of perovskite-based solar cells was based on monohalide material. ${ }^{15}$ In this case, the power conversion efficiency is usually limited to less than $20 \%$ for conventional $\mathrm{MAPbI}_{3}$-based devices, ${ }^{16}$ which are additionally plagued by poor resistance to moisture or high temperatures, ${ }^{17,18}$ as well as by the formation of trap states induced by exposure to light. ${ }^{19}$ The synthesis of $\mathrm{FAPbI}_{3}$ provides, in principle, an attractive alternative as it has a band gap smaller than its MA counterpart, ${ }^{20}$ being closer to the optimal value for a singlejunction solar cell, which influences favorably its conversion efficiency. ${ }^{1,21}$ However, the large radius of the FA cation favors the formation of a photoinactive polymorph at room temperature. $^{22}$ Alternatively, fully inorganic cesium-based $\mathrm{CsPbX}_{3}$ perovskite compounds ${ }^{23}$ with excellent thermal stability up to $450{ }^{\circ} \mathrm{C}^{24,25}$ have been explored as light harvesters. $^{26-28}$ Their use is partially hindered by the large band gap in the case of $\mathrm{CsPbBr}_{3}{ }^{27}$ and by the high-temperature formation of the photoactive polymorph, stable in ambient conditions for the lower band gap $\mathrm{CsPbI}_{3} .{ }^{23}$

The advantages of hybrid and inorganic perovskite families can be combined by introducing some amount of lighter inorganic cations into the organic lattice. Small molar fractions of $\mathrm{Cs}$ act as a crystallizer for the photoactive $\mathrm{FAPbI}_{3}$ phase, ${ }^{29,30}$ also reducing halide phase segregation. ${ }^{31}$ The improvement of structural properties, resulting in superior morphology, ${ }^{2,31}$ is accompanied by record solar cell efficiencies and by

Received: May 16, 2017

Accepted: June 12, 2017

Published: June 12, 2017 
significantly improved stability under standard operation conditions. ${ }^{2,3}$ Alloyed Cs- and $\mathrm{FAPbI}_{3}$ (band gap $\approx 1.73 \mathrm{eV}$ ) has been employed in perovskite-silicon tandem solar cells, $^{28,30,31}$ while the lower band gap FA/MA mixtures containing very small molar fractions of cesium and rubidium have demonstrated record high efficiencies exceeding $22 \%$ in a single-junction architecture. ${ }^{2,3}$ Despite the impressive performance of mixed cation devices, very little is known about the fundamental electronic properties of these materials. Moreover, a detailed knowledge of the electronic structure of fully inorganic perovskites is a crucial element for understanding the impact of the inorganic cation on the electronic properties of mixed compounds. Quantities relevant for photovoltaic applications, such as the exciton binding energy, the presence of the phase transition, and the dielectric screening, have not as yet been experimentally investigated in $\mathrm{CsPbX}_{3}$.

In this work, we present the results of systematic studies of the electronic and optical properties of inorganic $\mathrm{CsPbX}_{3}$ layers, where $\mathrm{X}=\mathrm{Br}$, I, or an alloy of the two, which have been heat treated to be in the metastable cubic black perovskite phase. Temperature-dependent absorption measurements demonstrate no evidence for any phase transitions below 300 $\mathrm{K}$, in striking contrast to their organic counterparts. We determine the exciton binding energy $\left(R^{*}\right)$ and reduced mass $(\mu)$ by magneto-transmission spectroscopy. Similarly to previous results obtained for hybrid organic inorganic compounds, we find that both the exciton binding energy and reduced mass scale linearly with the band gap energy. ${ }^{32,33}$ The small binding energy of the exciton in $\mathrm{CsPbI}_{3}$ compared to the thermal energy at room temperature suggests that in typical operational conditions for solar cells photocreated species exhibit a free-carrier-like behavior, which makes $\mathrm{CsPbI}_{3}$ an excellent building block for stable, high-efficiency devices. ${ }^{2,3} \mathrm{On}$ the basis of the value of the binding energy, we have calculated the dielectric constant $\left(\varepsilon_{\text {eff }}\right)$ and compared it with the values for organic-inorganic counterparts. Interestingly, $\varepsilon_{\text {eff }}$ is comparable for all of the iodide compounds but decreases significantly for the bromides. This suggests that at low temperature, when the motion of the organic cations is frozen, ${ }^{34}$ the dielectric screening mechanism is essentially the same for both the inorganic and hybrid perovskites and is controlled by the lead halide cage.

Typical transmission spectra of $\mathrm{Cs}_{\mathrm{PbBr}}, \mathrm{CsPbI}_{2} \mathrm{Br}$, and $\mathrm{CsPbI}_{3}$, measured over a wide range of temperatures (4.2-270 $\mathrm{K})$, are presented in Figure $1 \mathrm{a}-\mathrm{c}$. We observe a consistent blue shift of the band edge absorption energy of $\mathrm{CsPb}_{3}$ through mixed $\mathrm{CsPb}_{2} \mathrm{Br}$ to $\mathrm{CsPbBr}_{3}$, highlighting the good tuneability of the band gap via the introduction of a heavier halide in the crystal. ${ }^{35}$ The transmission spectrum for each compound exhibits a single minimum at all temperatures, which blue shifts and broadens with increasing temperature. The detailed evolution of the band gap absorption energy with temperature is presented in Figure 1d. We note that the band gaps of all of the investigated samples exhibit a well-behaved monotonic dependence on the temperature. This is in stark contrast with organic-inorganic halide perovskites, where an increase in the band gap is observed at temperatures corresponding to the phase transitions to a lower-symmetry crystalline structure. ${ }^{32,33,36-39}$ This is a particularly significant result in the case of $\mathrm{CsPbI}_{3}$, which suggests that our sample preparation procedure (see Experimental Methods) preserves the photoactive perovskite phase of this compound even at cryogenic temperatures. Importantly, we have monitored the absorption
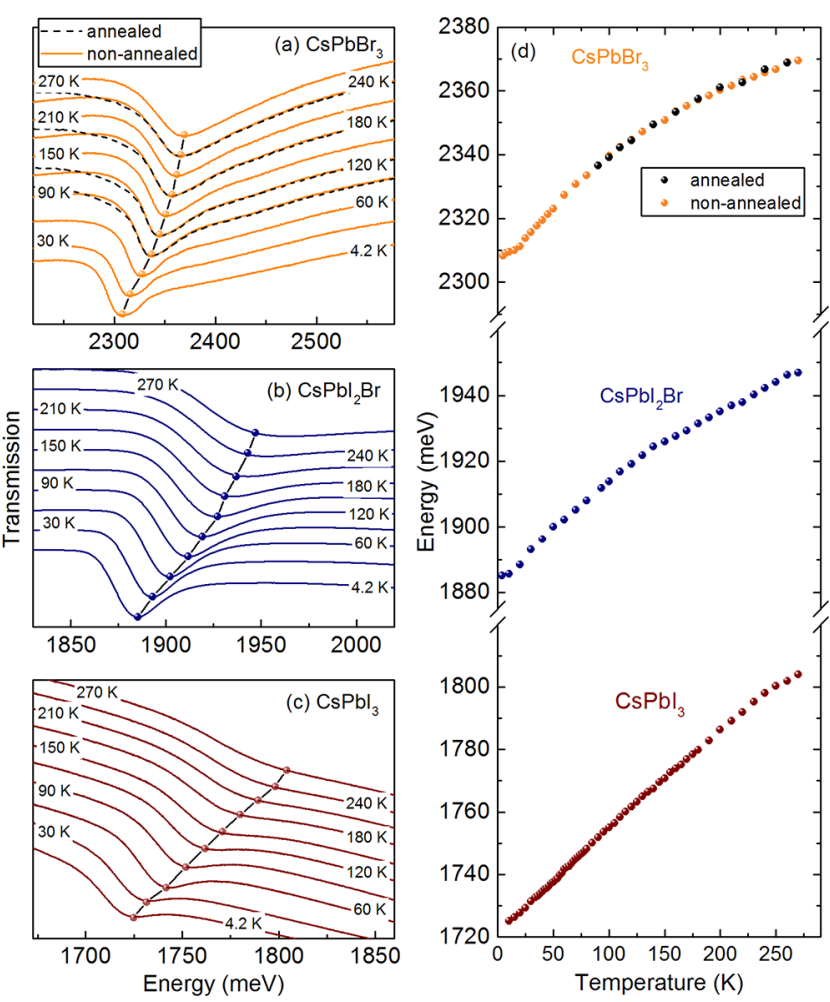

Figure 1. Transmission spectra measured at different temperatures for (a) $\mathrm{CsPbBr}_{3}$, (b) $\mathrm{CsPbI}_{2} \mathrm{Br}$, and (c) $\mathrm{CsPbI}_{3}$. Symbols highlight the evolution of the $1 \mathrm{~s}$ absorption with temperature. (d) Energy of the 1s transition (reflecting the evolution of the band gap) as a function of temperature for the three compounds. For $\mathrm{CsPbBr}_{3}$, data are shown for the as prepared and annealed samples as described in the text.

spectra of all of the samples during the cool down. This point was critical especially for the $\mathrm{CsPbI}_{3}$, which is known to be unstable at ambient conditions. In the absorption spectra, we did not see any signs of dramatic change of the band gap, which would suggest the transition into the yellow phase. This supports our finding that we freeze the sample in the cubic phase. In the case of $\mathrm{Cs}_{\mathrm{PbBr}}$, we have investigated both as prepared samples (orthorhombic phase) and samples that have been annealed at $250{ }^{\circ} \mathrm{C}$, which is above the transition to the cubic phase. ${ }^{27,40}$ The identical transmission data and evolution of the gap with temperature shown in Figure 1a,d demonstrate that annealing does not influence the electronic structure of $\mathrm{CsPbBr}_{3}$ under the measurement conditions used. This suggests that, independently of the thermal processing, $\mathrm{Cs} \mathrm{PbBr}_{3}$ will always transform to the orthorhombic phase below the phase transition point at $88{ }^{\circ} \mathrm{C}$, and the continuous evolution of its band gap with temperature indicates the absence of any further phase transitions and good stability of the investigated samples.

Low-temperature magneto-transmission spectroscopy is a powerful technique that has previously been used to precisely determine the binding energy and reduced mass of the exciton in organic-inorganic perovskites. ${ }^{32,33,39}$ In Figure 2a, we present typical magneto-transmission spectra of $\mathrm{Cs} \mathrm{PbBr}_{3}$ measured at $2 \mathrm{~K}$. Related magneto-transmission spectra of $\mathrm{CsPbI}_{3}$ and $\mathrm{CsPbI}_{2} \mathrm{Br}$ are shown in the Supporting Information (SI) (Figure S1). The pronounced minimum at $\sim 2313 \mathrm{meV}$ is attributed to the $1 \mathrm{~s}$ excitonic state. With increasing magnetic field, we observe a clear blue shift of this transition. On the 

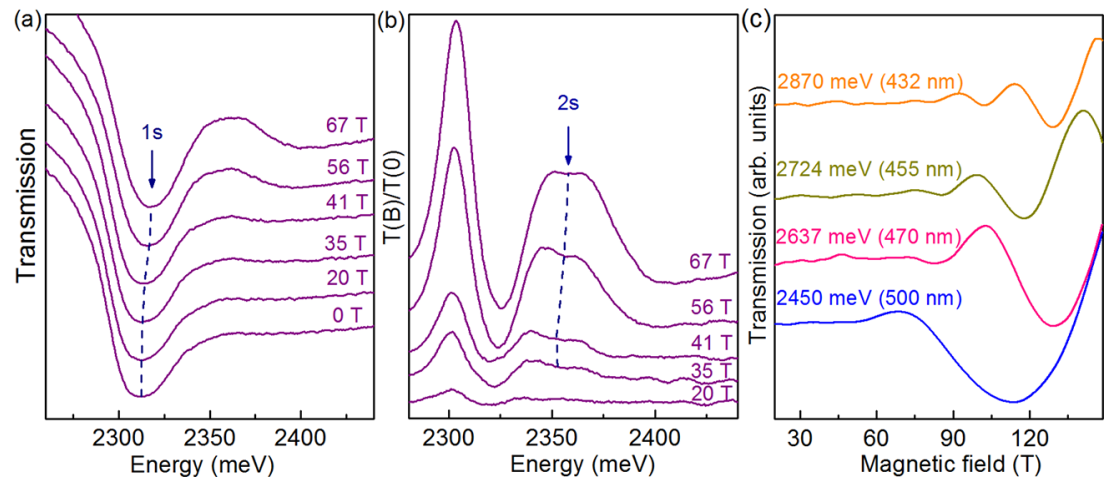

Figure 2. Low-temperature transmission spectra in a magnetic field for $\mathrm{CsPbBr}_{3}$. (a) Transmission spectra measured at the indicated magnetic field values. (b) Transmission spectra in the magnetic field divided by the zero field spectrum. (c) Monochromatic transmission as a function of magnetic field obtained by the short pulse technique.
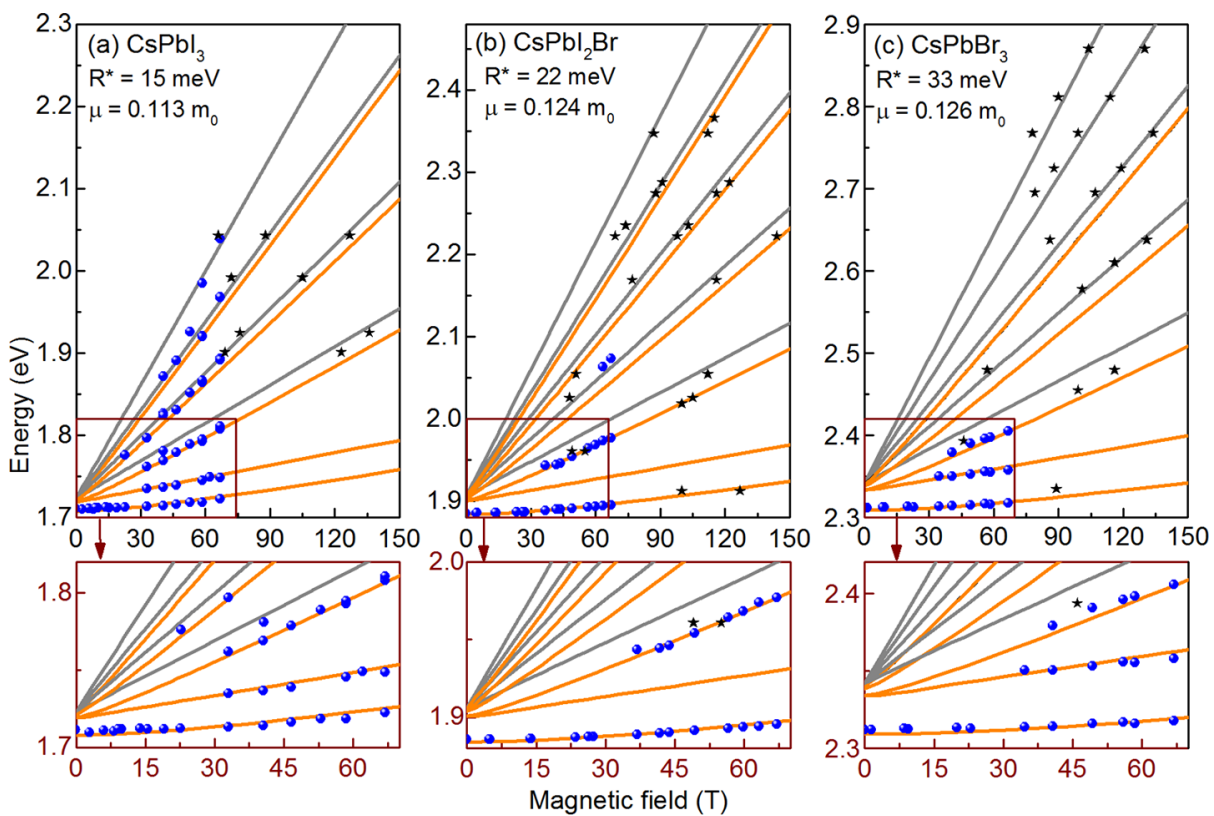

Figure 3. Energy fan chart. Excitonic transition and inter-Landau level transition energies as a function of magnetic field at $2 \mathrm{~K}_{\text {for }}(\mathrm{a}) \mathrm{CsPbI}_{3}$, (b) $\mathrm{CsPbI}_{2} \mathrm{Br}$, and (c) $\mathrm{CsPbBr}_{3}$. Orange lines are the results of the fit to hydrogen-like transitions. Gray lines indicate fitting result of the interband transition between Landau levels. Circles are data from long pulse field measurements, and stars are data from single-turn short pulse measurements. The lower panels show an expanded view of the low-field and low-energy portion of the fan chart.

high-energy side of the $1 \mathrm{~s}$ transition, a weak minimum can be resolved for magnetic fields larger than $35 \mathrm{~T}$. It is more clearly seen in differential transmission spectra obtained by dividing the transmission spectra by the spectrum measured at zero magnetic field (see Figure $2 b$ ). In a higher energy range, there are some hints of absorption due to free carrier transitions between Landau levels in the valence and conduction bands. To better resolve the free carrier transitions, we have extended the long pulse measurements to higher magnetic fields $(B>70 \mathrm{~T})$ using the short pulse generated by a single turn coil (see the Experimental Methods section) to follow the absorption minima to higher energies. Representative magneto-transmission curves measured with a single turn coil are presented in Figure 2c. These curves show the evolution of the transmission through the sample of a monochromatic (laser) light of different wavelengths measured as a function of the magnetic field. The minima correspond to dipole-allowed transitions between Landau levels in the conduction and valence bands.
To model the data further, we plot the energetic positions of the minima observed in the absorption spectra as a function of the magnetic field, as marked by blue and black points in Figure 3. Analysis of the full magnetic field dependence of both hydrogenic and free carrier transitions, shown in Figure 3, enables us to extract the exciton binding energy and reduced mass. ${ }^{32,33,39}$ We introduce a dimensionless parameter $\gamma=\hbar \omega_{\mathrm{c}} /$ $2 R^{*}$, where $\omega_{c}=e B / \mu$ is the cyclotron frequency of charge carriers and $\mu^{-1}=m_{\mathrm{e}}^{-1}+m_{\mathrm{h}}^{-1}$ defines the exciton reduced mass. In the high magnetic field limit (corresponding to $\gamma>1$ ), the observed absorption resonances are related to free carrier interLandau level transitions with energies

$$
E=E_{\mathrm{g}}+\left(n+\frac{1}{2}\right) \hbar \omega_{\mathrm{c}}
$$

where $E_{\mathrm{g}}$ is the band gap and $n=0,1,2, \ldots$ is the orbital quantum number of the Landau levels in the conduction and valence bands. For dipole-allowed transitions $(\Delta n=0)$ and for a well-defined value of the band gap $E_{g}$, the only fitting 
parameter in eq 1 is the reduced mass $\mu$. Fitting the observed resonances to eq 1 (gray lines in Figure 3) allows us to determine the exciton reduced mass. The excitonic-like transitions close to the band edge are well-described with a numerical model for a hydrogen atom in high magnetic field. ${ }^{41}$ The eigenenergies of an excitonic system in zero field are given by

$$
E_{N}=E_{\mathrm{g}}-\frac{R^{*}}{N^{2}}
$$

where $E_{N}$ is the energy of the $N$ th excitonic level, $R^{*}=R_{0} \mu$ / $m_{0} \varepsilon_{\text {eff }}^{2}, R_{0}$ is the atomic Rydberg, $m_{0}$ is the free electron mass, and $\varepsilon_{\text {eff }}$ is the relative dielectric constant. The fit of the interLandau level transitions provides an accurate estimation of $\mu$, which in this second step is taken as a fixed parameter. This provides strong limits on the value of $R^{*}$, which is further constrained by the observation of the $2 \mathrm{~s}$ state, well resolved only in the high magnetic field both for $\mathrm{CsPb}_{3}$ and $\mathrm{CsPbBr}$. Contrary to early magneto-optical estimates of exciton binding energies of hybrid organic-inorganic perovskite, ${ }^{42,43}$ our approach does not require us to assume a value for the effective dielectric constant, which we can actually determine from eq 2 . The values of the effective mass and $R^{*}$ obtained for all three compounds are summarized in Table 1 and Figure 5a,b.

Table 1. Parameters Determined from the Fit of the Full Landau Fan Chart for Cesium-Based Compounds at $2 \mathrm{~K}$

\begin{tabular}{llcccr} 
compound & \multicolumn{1}{c}{ phase } & $\begin{array}{c}E_{\mathrm{g}} \\
(\mathrm{meV})\end{array}$ & $\begin{array}{c}R^{*} \\
(\mathrm{meV})\end{array}$ & $\mu\left(\mathrm{m}_{0}\right)$ & $\varepsilon_{\text {eff }}$ \\
$\mathrm{CsPbI}_{3}$ & cubic & 1723 & $15 \pm 1$ & $0.114 \pm 0.01$ & 10.0 \\
$\mathrm{CsPbI}_{2} \mathrm{Br}$ & cubic & 1906 & $22 \pm 3$ & $0.124 \pm 0.02$ & 8.6 \\
$\mathrm{CsPBr}_{3}$ & orthorhombic & 2342 & $33 \pm 1$ & $0.126 \pm 0.01$ & 7.3 \\
\hline
\end{tabular}

In the case of organic-inorganic perovskites, the phase transition to a higher-symmetry crystalline structure allows rotational motion of the organic cation, which enhances the dielectric screening and reduces the exciton binding energy. ${ }^{32-34,39}$ The lack of an abrupt change of the band gap observed here implies that inorganic perovskites do not undergo phase transitions up to room temperature. The absence of a structural change suggests that the exciton binding energy does not vary over the investigated temperature interval. To support this conclusion, we have performed magnetotransmission measurements of $\mathrm{CsPbBr}_{3}$ at $180 \mathrm{~K}$. We compare the magnetic field dependence of the $1 \mathrm{~s}$ transition energy at high and low temperatures in Figure 4. The temperatureinduced change in the band gap has been removed by plotting the high-temperature data on a different scale (right axis) but

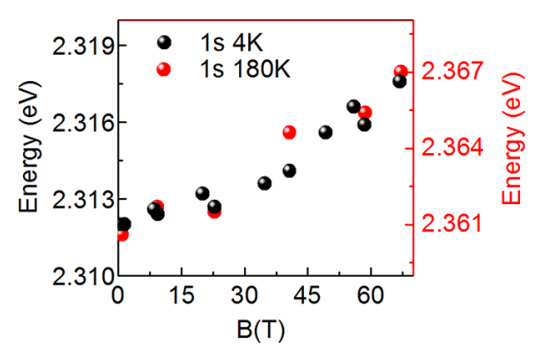

Figure 4. Magnetic field dependence of $1 \mathrm{~s}$ transition in $\mathrm{CsPbBr}_{3}$ at two different temperatures. over the same range ( $10 \mathrm{meV}$ in both cases). The excellent overlap of the two sets of data demonstrates that the exciton binding energy does not change within experimental accuracy.

In Figure 5b, we plot the experimentally determined values of the exciton reduced mass $\mu$ of cesium compounds. Our results

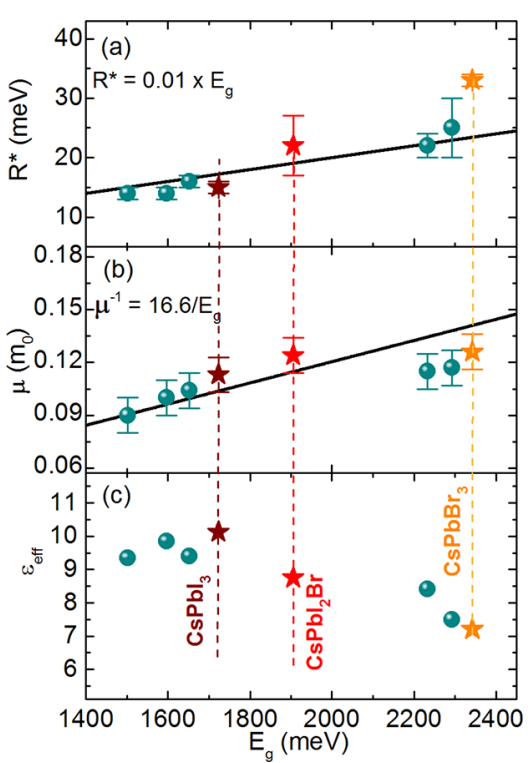

Figure 5. (a) Binding energy, (b) effective mass, and (c) dielectric constant as a function of the band gap. Brown, red, and yellow stars indicate the results for $\mathrm{CsPbI}_{3}, \mathrm{CsPbI}_{2} \mathrm{Br}$, and $\mathrm{CsPbBr}_{3}$, respectively. Blue symbols mark the results from previous work.

are close to theoretical predictions of $\mu$ for $\mathrm{CsPb}_{3}$, which range from $\sim 0.07 m_{0}{ }^{44,45}$ to $\sim 0.18 m_{0}{ }^{46}$ and in excellent agreement with density functional theory of organic-inorganic perovskites adjusted to fit the experimental band gap. ${ }^{47}$ We compare our results on fully inorganic compounds to the reduced masses determined on organic-inorganic perovskites. ${ }^{32,33,39} \mathrm{We}$ observe an increase of the effective mass with increasing value of the band gap. This trend can be understood in the frame of a simple two-band $\mathbf{k} \cdot \mathbf{p}$ model, ${ }^{48}$ and assuming the same effective mass for the valence and conduction band, the reduced mass of the exciton can be written as

$$
\frac{1}{\mu}=\frac{4|P|^{2}}{m_{0} E_{\mathrm{g}}}
$$

where $P=\left\langle\Psi_{\mathrm{VB}}\left|p_{x}\right| \Psi_{\mathrm{CB}}\right\rangle$ is the momentum matrix element that couples states in the conduction and valence bands and $2|P|^{2} /$ $m_{0}$ is the Kane energy. ${ }^{33}$ The measured evolution of the band gap with the reduced mass is well fitted with a Kane energy of $8.3 \mathrm{eV}$, only slightly larger than theoretical predictions. ${ }^{49}$

Knowing the values of the exciton binding energy and the reduced effective mass, we can calculate the effective dielectric

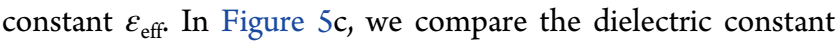
for cesium-based compounds (stars) with the corresponding quantities for the hybrid organic-inorganic materials from our previous work $^{32,33,39}$ (blue circles). The dielectric constant does not vary significantly for a given lead halide cage, regardless of the cation, while it decreases with decreasing halide mass. This is consistent with theoretical calculations of the complex dielectric function of MA- and $\mathrm{CsPbI}_{3}{ }^{50}$ and with the computed dielectric functions of $\mathrm{MASnX}_{3}{ }^{51}$ and $\mathrm{MAPbX}_{3}{ }^{52}$ which suggest that the main contributions to the dielectric permittivity 
are due to $\mathrm{Pb}-\mathrm{X}$ stretching modes and $\mathrm{Pb}-\mathrm{X}-\mathrm{Pb}$ rocking modes. ${ }^{53}$ Detailed Raman spectroscopy on the $\mathrm{MAPbX}_{3}$ series (with $\mathrm{X}=\mathrm{I}, \mathrm{Br}, \mathrm{Cl}$ ) has demonstrated that both $\mathrm{Pb}-\mathrm{X}$ stretching and $\mathrm{Pb}-\mathrm{X}-\mathrm{Pb}$ rocking modes harden with decreasing halide mass, with the derived dielectric function exhibiting the same trend as observed in Figure 5a. ${ }^{54}$ This confirms the qualitative picture that the dielectric screening properties are mainly determined by the lead halide cage in the low-temperature range, when the organic cation motions are frozen.

The exciton binding energies that we have determined are significantly smaller than early estimates obtained from magneto-optical measurements of the 1 s excitonic states. ${ }^{42}$ In the absence of any observed phase transitions (see Figure 1), we argue that the exciton binding energy will depend only very weakly on the temperature, as demonstrated by the similar diamagnetic shift of the $1 \mathrm{~s}$ state at 2 and $170 \mathrm{~K}$ in Figure 4 . This suggests that the photocreated carriers in the compounds investigated exhibit essentially free carrier behavior at temperatures corresponding to the normal operating conditions of solar cells.

In summary, we have presented a detailed magneto-optical investigation of fully inorganic $\mathrm{CsPbX}_{3}$ perovskites $(\mathrm{X}=\mathrm{I}, \mathrm{Br}$, and a mixture of these). The well-behaved (continuous) temperature dependence of the band gap points to the absence of any structural phase transitions for perovskites with fully inorganic cations. By performing magneto-transmission spectroscopy up to $150 \mathrm{~T}$, we have determined key electronic parameters including the exciton binding energy and reduced mass with high accuracy. Our approach does not require any assumption concerning the strength of the dielectric screening, which we determine a posteriori. The comparison of the values of the dielectric constant for inorganic and hybrid perovskites suggests that the dominant contribution to dielectric screening is related to the relative motion within the lead halide cage. For the fully inorganic compounds, the values of $R^{*}$ and $\mu$ increase with the band gap energy, similarly to hybrid perovskites. We conclude that at low temperature, when the organic cations are ordered, the qualitative picture of the interactions within the lattice is essentially the same for both the inorganic and hybrid compounds. This is consistent with the ability to optimize perovskite solar cells by adjusting the cation structure while maintaining the same device operation modes.

\section{EXPERIMENTAL METHODS}

Magneto-transmission spectra have been acquired by combining long pulse magnetic field measurements for magnetic fields up to $66 \mathrm{~T}$ and short duration pulsed magnets (for magnetic fields up to $150 \mathrm{~T}$ ). For the long pulse measurements (typical pulse duration $\approx 100 \mathrm{~ms}$ ), the sample was mounted in a liquid helium cryostat. White light from a halogen lamp was used as the excitation source. The light emitted from the lamp was coupled in a $200 \mu \mathrm{m}$ diameter multimode fiber, used to illuminate the sample. The transmitted light was coupled in a $400 \mu \mathrm{m}$ diameter multimode fiber and guided to a spectrometer equipped with a liquid-nitrogen-cooled CCD camera. The typical exposure time was $2 \mathrm{~ms}$, which ensured that the transmission spectra were acquired at essentially constant magnetic field values. For the very high magnetic field measurements $(B<150 \mathrm{~T})$, magnetic field pulses with a typical duration of $5 \mu \mathrm{s}$ were generated by a single turn coil system with a bore diameter of $10 \mathrm{~mm}$. A helium flow cryostat with a kapton tail was located in the single turn coil. The sample was kept at $5 \mathrm{~K}$. Magneto-transmission measurements were conducted by using a tunable optical parametric oscillator (OPO) pumped by a Ti:sapphire laser as the light source, a fast $(100 \mathrm{MHz})$ silicon detector, and a high-speed digital oscilloscope.

All samples were prepared on glass microscope slides, which were cleaned by sonication sequentially in acetone and isopropanol, and then treated with oxygen plasma for $10 \mathrm{~min}$. The $\mathrm{CsPbI}_{3}$ and $\mathrm{CsPbI}_{2} \mathrm{Br}$ perovskite layers were deposited in a nitrogen glovebox by spin-coating (at $1500 \mathrm{rpm}$ ) a solution of the appropriate ratios of CsI (Alfa Aesar, 99.9\%), $\mathrm{PbI}_{2}$ (SigmaAldrich, 99\%), and $\mathrm{PbBr}_{2}$ (Sigma-Aldrich, > 98\%) dissolved at $0.43 \mathrm{M}$ in $\mathrm{N}, \mathrm{N}$-dimethylformamide following a previously reported procedure. ${ }^{35}$ The $\mathrm{CsPBr}_{3}$ perovskite was deposited by sequential evaporation of layers of $\mathrm{PbBr}_{2}(107 \mathrm{~nm})$ and $\mathrm{CsBr}(93 \mathrm{~nm})$, each deposited at $1-2 \AA / \mathrm{s}$ onto roomtemperature substrates, at pressures below $6 \times 10^{-6} \mathrm{mbar}$ in a BOC Edwards Auto 306 evaporator. Owing to their limited stability in air at room temperature, $\mathrm{CsPbX}_{3}$ samples were systematically annealed in an oven before loading them in the cryostat in order to restore the cubic phase. $\mathrm{CsPbI}_{3}$ was annealed at $350{ }^{\circ} \mathrm{C}$ for $10 \mathrm{~min} . \mathrm{CsPb}_{2} \mathrm{Br}$ and $\mathrm{CsPbr}_{3}$ were annealed at $250{ }^{\circ} \mathrm{C}$ for 5-7 $\mathrm{min}$. After the annealing, the samples were placed in a liquid helium cryostat within $4 \mathrm{~min}$, which ensured that the sample remained in the cubic phase. The sample stage was not temperature-controlled. All samples were annealed after deposition and again before measurement.

\section{ASSOCIATED CONTENT}

\section{Supporting Information}

The Supporting Information is available free of charge on the ACS Publications website at DOI: 10.1021/acsenergylett.7b00416.

Low-temperature transmission spectra in a magnetic field for $\mathrm{CsPbI}_{2} \mathrm{Br}$ and $\mathrm{CsPbI}_{3}, \mathrm{XRD}$, and absorbance and $\mathrm{PL}$ for $\mathrm{CsPbI}_{3} \mathrm{CsPbI}_{2} \mathrm{Br}$, and $\mathrm{CsPbr}_{3}$ (PDF)

\section{AUTHOR INFORMATION}

\section{Corresponding Authors}

*E-mail: paulina.plochocka@lncmi.cnrs.fr (P.P.).

*E-mail: robin.nicholas@physics.ox.ac.uk (R.J.N.).

ORCID *

R. J. Sutton: 0000-0002-6252-0840

P. Plochocka: 0000-0002-4019-6138

\section{Notes}

The authors declare no competing financial interest.

\section{ACKNOWLEDGMENTS}

This work was partially supported by the ANR JCJC project milliPICS, the Région Midi-Pyrénées under Contract MESR 13053031, the BLAPHENE and TERASPEC project, which received funding from the IDEX Toulouse, and the Emergence program "Programme des Investissements d'Avenir" under the Program ANR-11-IDEX-0002-02, Reference ANR-10-LABX0037-NEXT. Z.Y. held a fellowship from the Chinese Scholarship Council (CSC), R.J.S. is a Commonwealth Scholar, funded by the U.K. government. This work was supported by EPSRC (U.K.) via its membership to the EMFL (Grant No. $\mathrm{EP} / \mathrm{N} 01085 \mathrm{X} / 1)$. 


\section{REFERENCES}

(1) Yang, W. S.; Noh, J. H.; Jeon, N. J.; Kim, Y. C.; Ryu, S.; Seo, J.; Seok, S. I. High-Performance Photovoltaic Perovskite Layers Fabricated through Intramolecular Exchange. Science 2015, 348, 1234-1237.

(2) Saliba, M.; Matsui, T.; Seo, J.-Y.; Domanski, K.; Correa-Baena, J.P.; Nazeeruddin, M. K.; Zakeeruddin, S. M.; Tress, W.; Abate, A.; Hagfeldt, A.; et al. Cesium-Containing Triple Cation Perovskite Solar Cells: Improved Stability, Reproducibility and High Efficiency. Energy Environ. Sci. 2016, 9, 1989-1997.

(3) Saliba, M.; Matsui, T.; Domanski, K.; Seo, J.-Y.; Ummadisingu, A.; Zakeeruddin, S. M.; Correa-Baena, J.-P.; Tress, W. R.; Abate, A.; Hagfeldt, A.; et al. Incorporation of Rubidium Cations into Perovskite Solar Cells Improves Photovoltaic Performance. Science 2016, 354, 206-209.

(4) Shin, S. S.; Yeom, E. J.; Yang, W. S.; Hur, S.; Kim, M. G.; Im, J.; Seo, J.; Noh, J. H.; Seok, S. I. Colloidally Prepared La-Doped $\mathrm{BaSnO}_{3}$ Electrodes for Efficient, Photostable Perovskite Solar Cells. Science 2017, 356, 167-171.

(5) Burschka, J.; Pellet, N.; Moon, S.-J.; Humphry-Baker, R.; Gao, P.; Nazeeruddin, M. K.; Grätzel, M. Sequential Deposition as a Route to High-Performance Perovskite-Sensitized Solar Cells. Nature 2013, 499, 316-319.

(6) Liu, M.; Johnston, M. B.; Snaith, H. J. Efficient Planar Heterojunction Perovskite Solar Cells by Vapour Deposition. Nature 2013, 501, 395-398.

(7) Tanaka, K.; Takahashi, T.; Ban, T.; Kondo, T.; Uchida, K.; Miura, N. Comparative Study on the Excitons in Lead-Halide-Based Perovskite-Type Crystals $\mathrm{CH}_{3} \mathrm{NH}_{3} \mathrm{PbBr}_{3} \mathrm{CH}_{3} \mathrm{NH}_{3} \mathrm{PbI}_{3}$. Solid State Commun. 2003, 127, 619-623.

(8) Deschler, F.; et al. High Photoluminescence Efficiency and Optically Pumped Lasing in Solution-Processed Mixed Halide Perovskite Semiconductors. J. Phys. Chem. Lett. 2014, 5, 1421-1426.

(9) Huang, H.; Susha, A. S.; Kershaw, S. V.; Hung, T. F.; Rogach, A. L. Control of Emission Color of High Quantum Yield $\mathrm{CH}_{3} \mathrm{NH}_{3} \mathrm{PbBr}_{3}$ Perovskite Quantum Dots by Precipitation Temperature. Adv. Sci. 2015, 2, 1500194.

(10) Stranks, S. D.; Eperon, G. E.; Grancini, G.; Menelaou, C.; Alcocer, M. J.; Leijtens, T.; Herz, L. M.; Petrozza, A.; Snaith, H. J. Electron-Hole Diffusion Lengths Exceeding 1 Micrometer in an Organometal Trihalide Perovskite Absorber. Science 2013, 342, 341344.

(11) Xing, G.; Mathews, N.; Sun, S.; Lim, S. S.; Lam, Y. M.; Grätzel, M.; Mhaisalkar, S.; Sum, T. C. Long-Range Balanced Electron- and Hole-Transport Lengths in Organic-Inorganic $\mathrm{CH}_{3} \mathrm{NH}_{3} \mathrm{PbI}_{3}$. Science 2013, 342, 344-347.

(12) Tan, Z.-K.; et al. Bright Light-Emitting Diodes Based on Organometal Halide Perovskite. Nat. Nanotechnol. 2014, 9, 687-692.

(13) Zhu, H.; Fu, Y.; Meng, F.; Wu, X.; Gong, Z.; Ding, Q.; Gustafsson, M. V.; Trinh, M. T.; Jin, S.; Zhu, X. Lead Halide Perovskite Nanowire Lasers with Low Lasing Thresholds and High Quality Factors. Nat. Mater. 2015, 14, 636-642.

(14) Fang, Y.; Dong, Q.; Shao, Y.; Yuan, Y.; Huang, J. Highly Narrowband Perovskite Single-Crystal Photodetectors Enabled by Surface-Charge Recombination. Nat. Photonics 2015, 9, 679-686.

(15) Correa-Baena, J.-P.; Abate, A.; Saliba, M.; Tress, W.; Jacobsson, T. J.; Grätzel, M.; Hagfeldt, A. The Rapid Evolution of Highly Efficient Perovskite Solar Cells. Energy Environ. Sci. 2017, 10, 710-727.

(16) Roldan-Carmona, C.; Gratia, P.; Zimmermann, I.; Grancini, G.; Gao, P.; Graetzel, M.; Nazeeruddin, M. K. High Efficiency Methylammonium Lead Triiodide Perovskite Solar Cells: the Relevance of Non-Stoichiometric Precursors. Energy Environ. Sci. 2015, 8, 3550-3556.

(17) Leijtens, T.; Eperon, G. E.; Noel, N. K.; Habisreutinger, S. N.; Petrozza, A.; Snaith, H. J. Stability of Metal Halide Perovskite Solar Cells. Adv. Energy Mater. 2015, 5, 1500963 . , and others cited there.

(18) Misra, R. K.; Aharon, S.; Li, B.; Mogilyansky, D.; Visoly-Fisher, I.; Etgar, L.; Katz, E. A. Temperature-and Component-Dependent
Degradation of Perovskite Photovoltaic Materials under Concentrated Sunlight. J. Phys. Chem. Lett. 2015, 6, 326-330.

(19) Hoke, E. T.; Slotcavage, D. J.; Dohner, E. R.; Bowring, A. R.; Karunadasa, H. I.; McGehee, M. D. Reversible Photo-Induced Trap Formation in Mixed-Halide Hybrid Perovskites for Photovoltaics. Chem. Sci. 2015, 6, 613-617.

(20) Koh, T. M.; Fu, K.; Fang, Y.; Chen, S.; Sum, T.; Mathews, N.; Mhaisalkar, S. G.; Boix, P. P.; Baikie, T. Formamidinium-Containing Metal-Halide: An Alternative Material for Near-IR Absorption Perovskite Solar Cells. J. Phys. Chem. C 2014, 118, 16458-16462.

(21) Lee, J.-W.; Seol, D.-J.; Cho, A.-N.; Park, N.-G. High-Efficiency Perovskite Solar Cells Based on the Black Polymorph of HC$\left(\mathrm{NH}_{2}\right)_{2} \mathrm{PbI}_{3}$. Adv. Mater. 2014, 26, 4991-4998.

(22) Li, Z.; Yang, M.; Park, J.-S.; Wei, S.-H.; Berry, J. J.; Zhu, K. Stabilizing Perovskite Structures by Tuning Tolerance Factor: Formation of Formamidinium and Cesium Lead Iodide Solid-State Alloys. Chem. Mater. 2016, 28, 284-292.

(23) Møller, C. K. Crystal Structure and Photoconductivity of Cæsium Plumbohalides. Nature 1958, 182, 1436-1436.

(24) da Silva, E. L.; Skelton, J. M.; Parker, S. C.; Walsh, A. Phase Stability and Transformations in the Halide Perovskite CsSnI ${ }_{3}$. Phys. Rev. B: Condens. Matter Mater. Phys. 2015, 91, 144107.

(25) Kulbak, M.; Gupta, S.; Kedem, N.; Levine, I.; Bendikov, T.; Hodes, G.; Cahen, D. Cesium Enhances Long-Term Stability of Lead Bromide Perovskite-Based Solar Cells. J. Phys. Chem. Lett. 2016, 7, $167-172$.

(26) Eperon, G. E.; Paternò, G. M.; Sutton, R. J.; Zampetti, A.; Haghighirad, A. A.; Cacialli, F.; Snaith, H. J. Inorganic Caesium Lead Iodide Perovskite Solar Cells. J. Mater. Chem. A 2015, 3, 1968819695.

(27) Kulbak, M.; Cahen, D.; Hodes, G. How Important Is the Organic Part of Lead Halide Perovskite Photovoltaic Cells? Efficient CsPbBr ${ }_{3}$ Cells. J. Phys. Chem. Lett. 2015, 6, 2452-2456.

(28) Beal, R. E.; Slotcavage, D. J.; Leijtens, T.; Bowring, A. R.; Belisle, R. A.; Nguyen, W. H.; Burkhard, G. F.; Hoke, E. T.; McGehee, M. D. Cesium Lead Halide Perovskites with Improved Stability for Tandem Solar Cells. J. Phys. Chem. Lett. 2016, 7, 746-751.

(29) Yi, C.; Luo, J.; Meloni, S.; Boziki, A.; Ashari-Astani, N.; Grätzel, C.; Zakeeruddin, S. M.; Röthlisberger, U.; Grätzel, M. Entropic Stabilization of Mixed A-Cation $\mathrm{ABX}_{3}$ Metal Halide Perovskites for High Performance Perovskite Solar Cells. Energy Environ. Sci. 2016, 9, $656-662$.

(30) Lee, J.-W.; Kim, D.-H.; Kim, H.-S.; Seo, S.-W.; Cho, S. M.; Park, N.-G. Formamidinium and Cesium Hybridization for Photo- and Moisture-Stable Perovskite Solar Cell. Adv. Energy Mater. 2015, 5, 1501310.

(31) McMeekin, D. P.; et al. A Mixed-Cation Lead Mixed-Halide Perovskite Absorber for Tandem Solar Cells. Science 2016, 351, 151155 .

(32) Miyata, A.; Mitioglu, A.; Plochocka, P.; Portugall, O.; Wang, J. T.-W.; Stranks, S. D.; Snaith, H. J.; Nicholas, R. J. Direct Measurement of the Exciton Binding Energy and Effective Masses for Charge Carriers in an Organic-Inorganic Tri-halide Perovskite. Nat. Phys. 2015, 11, 582-587.

(33) Galkowski, K.; et al. Determination of the Exciton Binding Energy and Effective Masses for Methylammonium and Formamidinium Lead Tri-Halide Perovskite Semiconductors. Energy Environ. Sci. 2016, 9, 962-970.

(34) Poglitsch, A.; Weber, D. Dynamic Disorder in Methylammoniumtrihalogenplumbates (II) Observed by Millimeter Wave Spectroscopy. J. Chem. Phys. 1987, 87, 6373-6378.

(35) Sutton, R. J.; et al. Bandgap-Tunable Cesium Lead Halide Perovskites with High Thermal Stability for Efficient Solar Cells. Adv. Energy Mater. 2016, 6, 1502458.

(36) Yamada, Y.; Nakamura, T.; Endo, M.; Wakamiya, A.; Kanemitsu, Y. Photoelectronic Responses in Solution-Processed Perovskite $\mathrm{CH}_{3} \mathrm{NH}_{3} \mathrm{PbI}_{3}$ Solar Cells Studied by Photoluminescence and Photoabsorption Spectroscopy. IEEE J. Photovolt 2015, 5, 401-405. 
(37) D’Innocenzo, V.; Grancini, G.; Alcocer, M. J.; Kandada, A. R. S.; Stranks, S. D.; Lee, M. M.; Lanzani, G.; Snaith, H. J.; Petrozza, A. Excitons Versus Free Charges in Organo-Lead Tri-Halide Perovskites. Nat. Commun. 2014, 5, 3586.

(38) Galkowski, K.; et al. Spatially Resolved Studies of the Phases and Morphology of Methylammonium and Formamidinium Lead TriHalide Perovskites. Nanoscale 2017, 9, 3222.

(39) Yang, Z.; Surrente, A.; Galkowski, K.; Bruyant, N.; Maude, D. K.; Haghighirad, A. A.; Snaith, H. J.; Plochocka, P.; Nicholas, R. J. Unraveling the Exciton Binding Energy and the Dielectric Constant in Single-Crystal Methylammonium Lead Triiodide Perovskite. J. Phys. Chem. Lett. 2017, 8, 1851-1855.

(40) Stoumpos, C. C.; et al. Crystal Growth of the Perovskite Semiconductor $\mathrm{CsPbBr}_{3}$ : A New Material for High-Energy Radiation Detection. Cryst. Growth Des. 2013, 13, 2722-2727.

(41) Makado, P. C.; McGill, N. C. Energy Levels of a Neutral Hydrogen-Like System in a Constant Magnetic Field of Arbitrary Strength. J. Phys. C: Solid State Phys. 1986, 19, 873.

(42) Hirasawa, M.; Ishihara, T.; Goto, T.; Uchida, K.; Miura, N. Magnetoabsorption of the Lowest Exciton in Perovskite-Type Compound $\left(\mathrm{CH}_{3} \mathrm{NH}_{3}\right) \mathrm{PbI}_{3}$. Phys. B 1994, 201, 427-430.

(43) Tanaka, K.; Takahashi, T.; Ban, T.; Kondo, T.; Uchida, K.; Miura, N. Comparative Study on the Excitons in Lead-Halide-Based Perovskite-Type Crystals $\mathrm{CH}_{3} \mathrm{NH}_{3} \mathrm{PbBr}_{3} \mathrm{CH}_{3} \mathrm{NH}_{3} \mathrm{PbI}_{3}$. Solid State Commun. 2003, 127, 619-623.

(44) Chang, Y.; Park, C.; Matsuishi, K. First-Principles Study of the Structural and the Electronic Properties of the Lead-Halide-Based Inorganic-Organic Perovskites $\left(\mathrm{CH}_{3} \mathrm{NH}_{3}\right) \mathrm{PbX}_{3}$ and $\mathrm{CsPbX}(\mathrm{X}=\mathrm{Cl}$, Br, I). J. Korean Phys. Soc. 2004, 44, 889-893.

(45) Amat, A.; Mosconi, E.; Ronca, E.; Quarti, C.; Umari, P.; Nazeeruddin, M. K.; Grätzel, M.; De Angelis, F. Cation-Induced BandGap Tuning in Organohalide Perovskites: Interplay of Spin-Orbit Coupling and Octahedra Tilting. Nano Lett. 2014, 14, 3608-3616.

(46) Giorgi, G.; Fujisawa, J.-I.; Segawa, H.; Yamashita, K. Cation Role in Structural and Electronic Properties of 3D Organic-Inorganic Halide Perovskites: a DFT Analysis. J. Phys. Chem. C 2014, 118, 12176-12183.

(47) Umari, P.; Mosconi, E.; De Angelis, F. Relativistic GW Calculations on $\mathrm{CH}_{3} \mathrm{NH}_{3} \mathrm{PbI}_{3}$ and $\mathrm{CH}_{3} \mathrm{NH}_{3} \mathrm{SnI}_{3}$ Solar Cells. Sci. Rep. 2015, 4, 4467.

(48) Even, J.; Pedesseau, L.; Katan, C.; Kepenekian, M.; Lauret, J.-S.; Sapori, D.; Deleporte, E. Solid-State Physics Perspective on Hybrid Perovskite Semiconductors. J. Phys. Chem. C 2015, 119, 10161-10177.

(49) Even, J.; Pedesseau, L.; Katan, C. Analysis of Multivalley and Multibandgap Absorption and Enhancement of Free Carriers Related to Exciton Screening in Hybrid Perovskites. J. Phys. Chem. C 2014, 118, 11566-11572.

(50) Berdiyorov, G. R.; Kachmar, A.; El-Mellouhi, F.; Carignano, M. A.; El-Amine Madjet, M. Role of Cations on the Electronic Transport and Optical Properties of Lead-Iodide Perovskites. J. Phys. Chem. C 2016, 120, 16259-16270.

(51) Feng, J.; Xiao, B. Effective Masses and Electronic and Optical Properties of Nontoxic $\mathrm{MASnX}_{3}(\mathrm{X}=\mathrm{Cl}, \mathrm{Br}$, and I) Perovskite Structures as Solar Cell Absorber: A Theoretical Study Using HSE06. J. Phys. Chem. C 2014, 118, 19655-19660.

(52) Feng, J.; Xiao, B. Crystal Structures, Optical Properties, and Effective Mass Tensors of $\mathrm{CH}_{3} \mathrm{NH}_{3} \mathrm{PbX}_{3}(\mathrm{X}=\mathrm{I}$ and $\mathrm{Br}$ ) Phases Predicted from HSE06. J. Phys. Chem. Lett. 2014, 5, 1278-1282.

(53) Pérez-Osorio, M. A.; Milot, R. L.; Filip, M. R.; Patel, J. B.; Herz, L. M.; Johnston, M. B.; Giustino, F. Vibrational Properties of the Organic-Inorganic Halide Perovskite $\mathrm{CH}_{3} \mathrm{NH}_{3} \mathrm{PbI}_{3}$ from Theory and Experiment: Factor Group Analysis, First-Principles Calculations, and Low-Temperature Infrared Spectra. J. Phys. Chem. C 2015, 119, 25703-25718.

(54) Sendner, M.; et al. Optical Phonons in Methylammonium Lead Halide Perovskites and Implications for Charge Transport. Mater. Horiz. 2016, 3, 613-620. 